\title{
Stability analysis of a variable meme transmission model
}

\author{
Reem Al-Amoudi, Salma Al-Tuwairqi*, Sarah Al-Sheikh \\ Department of mathematics, King Abdulaziz university, Jeddah 21551, Saudi Arabia. \\ *Corresponding author E-mail: saltuwairqi@kau.edu.sa
}

\begin{abstract}
Copyright (C) 2014 Reem Al-Amoudi et. al. This is an open access article distributed under the Creative Commons Attribution License Creative Commons Attribution License, which permits unrestricted use, distribution, and reproduction in any medium, provided the original work is properly cited.
\end{abstract}

\begin{abstract}
Memes propagation is a usual form of social interaction. Understanding the dynamics of memes transmission enables one to find the conditions that leads to persistence or disappearance of memes. In this paper we analyze qualitatively a mathematical model of variable meme transmission. Two equilibrium points of the model are examined: meme free equilibrium and meme existence equilibrium. The reproduction number $\mathcal{R}_{0}$ that generates new memes is found. Local and global stability of the equilibrium points are explored. Finally, we support our results using numerical simulations.
\end{abstract}

Keywords: Basic reproduction number, Global stability, Local stability, Liapunov function, Variable memes transmission model.

\section{Introduction}

Meme was first defined by Dawkins [1] as "a unit of cultural transmission, or a unit of immitation". Style of living, eating, clothing and thinking are all examples of memes. Transmission of memes occurs more frequently these days than ever before, due to easy tech communication between individuals. There is a strong resemblance between the propagation of memes and the spread of rumors. In addition, both may be regarded as a virus, that is transmitted to another individual by reading, hearing, seeing or any other way.

Researchers have applied epidemiological models to study the dynamics of social systems. In particular, the dynamics of rumors spread and transmission of ideas and thoughts. This was based on the fact that both biological diseases and social behavior are a result from interactions between individuals. Daley and kendall are among the earliest researchers to propose a rumor spread model that has some properties in common with epidemic model [2]. Also Cane [3] showed that deterministic forms of models for the spread of an epidemic and of a rumor are similar. At the beginning of this century, Thompson et al. [4] explored the dynamics of rumor spreading in chat rooms. Bettencourt et al. [5] applied models similar to epidemiology to the spread of ideas. Kawachi [6] proposed deterministic models for rumor transmission with constant and variable rumor in an age-independent population. Al-Amoudi et al. [7] analyzed qualitatively a meme propagation model of a constant meme. Kawachi et al. [8] explored the effects of various contact interactions in a rumor transmission model. Piqueira [9] examined an equilibrium study of a rumor spreading model according to propagation parameters and initial conditions. Huang [10] studied the rumor spreading process with denial and skepticism. Wang and Wood [11] adopted an epidemiological approach to model viral meme propagation. Huo et al. [12] analyzed the dynamics of a rumor 
transmission model with incubation. Zhao et al. [13, 14] proposed rumor spreading models in social networks considering the forgetting mechanism of spreaders.

Studying the dynamics of memes propagation gives an insight on ways to motivate public interest to a certain idea, change perception and persuade others.

In this paper, we analyze qualitatively a model of a variable meme transmission in a population with constant immigration and emigration. This model was proposed by Kawachi [6] in studying the propagation of variable rumors, which he defines as rumors being modified within the process of communication. He examined the limit system of the model, that is, reducing the model to a system of two ordinary differential equations. Here, we investigate and give a comprehensive qualitative study for the full system of the model. The mathematical model and basic reproduction number is described in section 2. Equilibria of the model are identified in section 3. Section 4 analyses the stability of equilibria both locally and globally using linearization methods and Lyapunov method. Numerical simulations are illustrated in section 5. Discussions and conclusions are given in Section 6.

\section{Variable meme model and basic reproduction number}

Let $\mathrm{N}(\mathrm{t})$ denote the total population. We divided the population into three disjoint classes of individuals: $\mathrm{S}(\mathrm{t})$, the susceptible class, describing individuals who have not yet been exposed to a particular meme; $\mathrm{I}(\mathrm{t})$, the spreader class, referring to individuals who have taken an active interest in the idea or concept that a meme represents, and therefore have a tendency to talk about the meme in social interactions; $\mathrm{Z}(\mathrm{t})$, the stifler class, meaning individuals who have experienced the meme, but have no interest or have lost interest at some point to spread it.

When a meme slips out of the memory of stiflers, or when a meme is modified in a way that is presented to a stifler as a new meme, then transition from stifler class to susceptible class takes place. Here, the meme is regarded as variable due to modification process. The variable meme model is governed by the following nonlinear system of ordinary differential equations:

$$
\begin{aligned}
\frac{d S}{d t} & =B+\eta Z-\alpha S I-\mu S, \\
\frac{d I}{d t} & =\alpha \theta S I-\beta I^{2}-\gamma I Z-\mu I, \\
\frac{d Z}{d t} & =\alpha(1-\theta) S I+\beta I^{2}+\gamma I Z-\eta Z-\mu Z,
\end{aligned}
$$

where the positive parameters are defined as follows: $B$ is the sum of the population's birth rate and immigration rate; $\mu$ is the sum of the population's death rate and emigration rate; $\alpha$ is the rate at which susceptibles change their meme class, where $\alpha=c q$ such that $c$ is the average number of contact per unit time and $q$ is the probability of transmitting the meme; $\beta$ is the rate at which spreaders become stiflers by contacting with each other; $\gamma$ is the rate at which spreaders become stiflers by contacting with stiflers; $\theta$ is the fraction of susceptibles who become spreaders (at rate $\alpha) ; 1-\theta$ is the fraction of susceptibles who become stiflers (at rate $\alpha$ ), such that $\theta \in(0,1]$; and $\eta$ is the rate at which stiflers become susceptibles again.

Note that $N(t)=S(t)+I(t)+Z(t)$. It follows from system (1) that $N^{\prime}(t)+\mu N(t)=B$, which has the solution $N(t)=N_{0} \exp (-\mu t)+[1-\exp (-\mu t)] B / \mu$, where $N_{0}=N(0)$, and therefore, $\lim _{t \rightarrow \infty} N(t)=B / \mu$. Thus, the considered region for system (1) is

$\Gamma=\left\{(S, I, Z): S+I+Z \leq \frac{B}{\mu}, S>0, I \geq 0, Z \geq 0\right\}$

The vector field points into the interior of $\Gamma$ on the part of its boundary when $S+I+Z=B / \mu$. Hence, $\Gamma$ is positively invariant.

The basic reproduction number $\mathcal{R}_{0}$ may be calculated by the method of next generation matrix [15]. First, we find the meme free equilibrium $E_{0}$, by equating the right hand side of system (1):

$$
\begin{aligned}
B+\eta Z-\alpha S I-\mu S & =0, \\
\alpha \theta S I-\beta I^{2}-\gamma I Z-\mu I & =0 \\
\alpha(1-\theta) S I+\beta I^{2}+\gamma I Z-\eta Z-\mu Z & =0 .
\end{aligned}
$$


The meme free equilibrium is $E_{0}=(B / \mu, 0,0)$. Let $X=(I, Z, S)^{T}$, then system (1) may be written as: $X^{\prime}=$ $\mathcal{F}(X)-\mathcal{V}(X)$ where

$$
\mathcal{F}(X)=\left[\begin{array}{c}
\alpha \theta S I \\
\alpha(1-\theta) S I \\
0
\end{array}\right], \mathcal{V}(X)=\left[\begin{array}{c}
\beta I^{2}+\gamma I Z+\mu I \\
-\beta I^{2}-\gamma I Z+\eta Z+\mu Z \\
-B-\eta Z+\alpha S I+\mu S
\end{array}\right]
$$

The Jacobian matrices of $\mathcal{F}(X)$ and $\mathcal{V}(X)$ at the meme free equilibrium point $E_{0}$, are

$$
\begin{gathered}
D \mathcal{F}\left(E_{0}\right)=\left[\begin{array}{ccc}
\alpha \theta S_{0} & 0 & 0 \\
\alpha(1-\theta) S_{0} & 0 & 0 \\
0 & 0 & 0
\end{array}\right]=\left[\begin{array}{cc}
F & 0 \\
0 & 0
\end{array}\right], \\
D \mathcal{V}\left(E_{0}\right)=\left[\begin{array}{ccc}
\mu & 0 & 0 \\
0 & \eta+\mu & 0 \\
\alpha S_{0} & 0 & \mu
\end{array}\right]=\left[\begin{array}{cc}
V & 0 \\
J_{1} & J_{2}
\end{array}\right],
\end{gathered}
$$

where $F=\left[\begin{array}{cc}\alpha \theta S_{0} & 0 \\ \alpha(1-\theta) S_{0} & 0\end{array}\right], V=\left[\begin{array}{cc}\mu & 0 \\ 0 & \eta+\mu\end{array}\right], J_{1}=\left[\begin{array}{ll}\alpha S_{0} & 0\end{array}\right], J_{2}=[\mu]$.

Thus the next generation matrix is $F V^{-1}=\left[\begin{array}{cc}\frac{\alpha \theta B}{\mu^{2}} & 0 \\ \frac{\alpha(1-\theta) B}{\mu^{2}} & 0\end{array}\right]$. Clearly, the spectral radius of matrix $F V^{-1}$ is $\rho\left(F V^{-1}\right)=\alpha \theta B / \mu^{2}$. So, the basic reproduction number of the system is $\mathcal{R}_{0}=\alpha \theta B / \mu^{2}$.

\section{Equilibria of the model}

Solution to system (2) gives two equilibrium points for model (1): the meme free equilibrium $E_{0}=(B / \mu, 0,0)$, and the positive (meme existence) equilibrium $E^{*}=\left(S^{*}, I^{*}, Z^{*}\right)$, where

$$
\begin{aligned}
S^{*} & =\frac{B+\eta Z^{*}}{\alpha I^{*}+\mu}=\frac{1}{\alpha I^{*}}\left[(\mu+\eta) Z^{*}+\mu I^{*}\right], \\
Z^{*} & =\frac{-\mu I^{*}}{\left(\alpha I^{*}+\mu\right)+\eta}\left[\frac{\alpha I^{*}}{\mu}+1-\frac{\alpha B}{\mu^{2}}\right] .
\end{aligned}
$$

So, if $\frac{\alpha B}{\mu^{2}} \leq 1$ then $Z^{*}<0$ which means that $Z^{*}$ does not exist biologically if $I^{*}>0$ and $\frac{\alpha B}{\mu^{2}} \leq 1$. Note that the condition $\frac{\alpha B}{\mu^{2}} \leq 1$ implies that $\mathcal{R}_{0}<1$.

Theorem 1 System (1) has two equilibria: the meme free equilibrium $E_{0}=(B / \mu, 0,0)$ which exists always; and the meme existence equilibrium $E^{*}=\left(S^{*}, I^{*}, Z^{*}\right)$ which does not exists if $\mathcal{R}_{0}<1$ and $I^{*}>0$.

\section{Stability of the equilibria}

\section{1. local stability}

Here we investigate the local stability of $E_{0}$ and $E^{*}$. We state the following theorems:

Theorem 2 (local stability of $\mathbf{E}_{0}$ ) If $\mathcal{R}_{0}<1$, the meme free equilibrium point $E_{0}$ is locally asymptotically stable. If $\mathcal{R}_{0}=1, E_{0}$ is locally stable. If $\mathcal{R}_{0}>1, E_{0}$ is unstable.

Proof. Linearizing system (1) (by linearization method [16]) we obtain the Jacobian matrix evaluated at the equilibrium $E_{0}=(B / \mu, 0,0)$ :

$J\left(E_{0}\right)=\left[\begin{array}{ccc}-\mu & \frac{-\alpha B}{\mu} & \eta \\ 0 & \frac{\alpha \theta B}{\mu}-\mu & 0 \\ 0 & \frac{\alpha(1-\theta) B}{\mu} & -\eta-\mu\end{array}\right]$. 
Clearly the roots of the characteristic equation are: $\lambda_{1}=-\mu<0, \lambda_{2}=-\eta-\mu<0$ and $\lambda_{3}=\frac{\alpha \theta B}{\mu}-\mu=$ $\mu\left(\frac{\alpha \theta B}{\mu^{2}}-1\right)=\mu\left(\mathcal{R}_{0}-1\right)<0$ if $\mathcal{R}_{0}<1$. Hence, $E_{0}$ is locally asymptotically stable if $\mathcal{R}_{0}<1$. If $\mathcal{R}_{0}=1$ then the eigenvalues are $\lambda_{1,2}=-\mu<0, \lambda_{3}=0$. So, $E_{0}$ is locally stable. If $\mathcal{R}_{0}>1$, then the characteristic equation has a positive eigenvalue. So, $E_{0}$ is unstable.

Theorem 3 (local stability of $\mathbf{E}^{*}$ ) The positive (meme existence) equilibrium $E^{*}$ is locally asymptotically stable if $\beta>\gamma$ and $\gamma \mu>\eta \alpha \theta$.

Proof. Linearizing system (1) at the equilibrium $E^{*}=\left(S^{*}, I^{*}, Z^{*}\right)$ gives

$J\left(E^{*}\right)=\left[\begin{array}{ccc}-\alpha I^{*}-\mu & -\alpha S^{*} & \eta \\ \alpha \theta I^{*} & \alpha \theta S^{*}-2 \beta I^{*}-\gamma Z^{*}-\mu & -\gamma I^{*} \\ \alpha(1-\theta) I^{*} & \alpha(1-\theta) S^{*}+2 \beta I^{*}+\gamma Z^{*} & \gamma I^{*}-\eta-\mu\end{array}\right]$

which is simplified using system (2) to be

$J\left(E^{*}\right)=\left[\begin{array}{ccc}\frac{-\left(B+\eta Z^{*}\right)}{S^{*}} & -\alpha S^{*} & \eta \\ \alpha \theta I^{*} & -\beta I^{*} & -\gamma I^{*} \\ \alpha(1-\theta) I^{*} & \beta I^{*}+\frac{(\mu+\eta) Z^{*}}{I^{*}} & \gamma I^{*}-\eta-\mu\end{array}\right]$.

The characteristic equation about $E^{*}$ is given by $\lambda^{3}+a_{1} \lambda^{2}+a_{2} \lambda+a_{3}=0$ where

$$
\begin{aligned}
& a_{1}=\mu+\eta+\frac{B+\eta Z^{*}}{S^{*}}+(\beta-\gamma) I^{*}>0 \text { if } \beta>\gamma \\
& a_{2}=\frac{\left(B+\eta Z^{*}\right) I^{*}}{S^{*}}(\beta-\gamma)+(\mu+\eta) \beta I^{*}+\gamma(\mu+\eta) Z^{*}+\frac{\mu\left(B+\eta Z^{*}\right)}{S^{*}}+\alpha^{2} \theta S^{*} I^{*}+\eta\left(\frac{\left(B+\eta Z^{*}\right)}{S^{*}}-\alpha I^{*}\right)+\eta \alpha \theta I^{*}>0
\end{aligned}
$$

if $\beta>\gamma$ where $\frac{\left(B+\eta Z^{*}\right)}{S^{*}}-\alpha I^{*}=\mu$.

$a_{3}=\eta \mu \beta I^{*}+\alpha S^{*} I^{*}\left[\eta \alpha \theta-\alpha \gamma I^{*}+\mu \alpha \theta\right]+\frac{\left(B+\eta Z^{*}\right) \beta \mu I^{*}}{S^{*}}+\gamma \alpha I^{*}(\mu+\eta) Z^{*}+[\gamma \mu-\eta \alpha \theta](\mu+\eta) Z^{*}$. Now, $S^{*}=\frac{\mu(\beta-\gamma) I^{*}+\mu^{2}+\eta \beta I^{*}+\mu \eta}{\alpha \mu \theta+\alpha \eta \theta-\gamma \alpha I^{*}}>0$. So, if $\beta>\gamma$ then $\alpha \mu \theta+\alpha \eta \theta-\gamma \alpha I^{*}>0$. Therefore, if $\beta>\gamma$ and $\gamma \mu>\eta \alpha \theta$ then $a_{3}>0$.

$$
\begin{aligned}
& a_{1} a_{2}-a_{3}=(\mu+\eta)\left[\frac{\left(B+\eta Z^{*}\right) I^{*}}{S^{*}}(\beta-\gamma)+(\mu+\eta) \beta I^{*}+\gamma(\mu+\eta) Z^{*}+\frac{\mu\left(B+\eta Z^{*}\right)}{S^{*}}+\eta \alpha \theta I^{*}+\eta \mu\right] \\
& +\frac{\left(B+\eta Z^{*}\right)}{S^{*}}\left[\frac{\left(B+\eta Z^{*}\right) I^{*}}{S^{*}}(\beta-\gamma)+\frac{\mu\left(B+\eta Z^{*}\right)}{S^{*}}+\eta \alpha \theta I^{*}+\alpha^{2} \theta S^{*} I^{*}+\eta \mu\right]+(\beta-\gamma) I^{*}\left[\frac{\left(B+\eta Z^{*}\right) I^{*}}{S^{*}}\right. \\
& \left.(\beta-\gamma)+(\mu+\eta) \beta I^{*}+\gamma(\mu+\eta) Z^{*}+\frac{\mu\left(B+\eta Z^{*}\right)}{S^{*}}+\alpha^{2} \theta S^{*} I^{*}+\eta \alpha \theta I^{*}+\eta \mu\right]+\eta \alpha \theta(\mu+\eta) Z^{*}+\eta \alpha \beta I^{* 2}
\end{aligned}
$$

$+\alpha^{2} \gamma S^{*} I^{* 2}>0$ if $\beta>\gamma$. Therefore if $\beta>\gamma$ and $\gamma \mu>\eta \alpha \theta$ then $a_{1} a_{2}-a_{3}>0, a_{1}>0$ and $a_{3}>0$. Hence, by using Routh-Herwitz Criteria [17], all eigenvalues of $J\left(E^{*}\right)$ have negative real parts. Thus, $E^{*}$ is locally asymptotically stable.

\subsection{Global Stability}

First, we explore the global stability of $E_{0}$. Consider the Lyapunov function [18]:

$$
\begin{aligned}
& L=I+Z, \\
& \frac{d L}{d t}=(-\mu+\alpha S) I-(\mu+\eta) Z .
\end{aligned}
$$

Since $E_{0} \in \Gamma$, then $S \leq \frac{B}{\mu}$ and we have

$$
\frac{d L}{d t} \leq\left(-\mu+\frac{\alpha B}{\mu}\right) I-(\mu+\eta) Z \leq 0 \text { if } \frac{\alpha B}{\mu} \leq \mu \text {. }
$$

Since $\mu$ and $\eta$ are non-negative, it follows that $\frac{d L}{d t}<0$ if $\frac{\alpha B}{\mu^{2}} \leq 1$; with $\frac{d L}{d t}=0$ if and only if $I=Z=0$.

Hence, the only solution of system (1) in $\Gamma$ on which $\frac{d L}{d t}=0$ is $E_{0}$. Therefore, by LaSalle's Invariance Principle [18], every solution of system (1), with initial conditions in $\Gamma$, approaches $E_{0}$ as $t \rightarrow \infty$. Hence, $E_{0}$ is globally asymptotically stable and we can state the following theorem. 
Theorem 4 (Global stability of $\mathbf{E}_{0}$ ) If $\frac{\alpha B}{\mu^{2}} \leq 1$ then $E_{0}$ is globally asymptotically stable in $\Gamma$.

Next, we examine the global stability of $E^{*}$. Consider the Lyapunov function:

$$
\begin{aligned}
& L=\frac{1}{2}\left[\left(S-S^{*}\right)+\left(I-I^{*}\right)+\left(Z-Z^{*}\right)\right]^{2}, \\
& \frac{d L}{d t}=\left[\left(S-S^{*}\right)+\left(I-I^{*}\right)+\left(Z-Z^{*}\right)\right][B-\mu S-\mu I-\mu Z] \\
& \frac{d L}{d t}=-\mu\left[\left(S-S^{*}\right)+\left(I-I^{*}\right)+\left(Z-Z^{*}\right)\right]^{2} \leq 0 .
\end{aligned}
$$

Since $B=\mu S^{*}+\mu I^{*}+\mu Z^{*}$. Hence, $E^{*}$ is globally stable and we can state the following theorem.

Theorem 5 (Global stability of $\mathbf{E}^{*}$ ) The positive (meme existence) equilibrium $E^{*}$ is globally stable.

We summarize the result of this section as follows:

- If $\mathcal{R}_{0}<1$, then $E_{0}$ is locally asymptotically stable. If $\frac{\alpha B}{\mu^{2}} \leq 1$ then $E_{0}$ is globally asymptotically stable and in this case $E^{*}$ does not exist.

- If $\beta>\gamma$ and $\gamma \mu>\eta \alpha \theta$ then $E^{*}$ is locally asymptotically stable. It is globally stable unconditionally whenever it exists.

\section{Numerical Simulation}

In this section, we illustrate numerical simulations of system (1) using different values of the parameters to support our results. Four different initial values are chosen such that $S+I+Z \leq B / \mu$ :

1. $\mathrm{S}(0)=0.3890, \mathrm{I}(0)=0.8540, \mathrm{Z}(0)=0.5360$,

2. $\mathrm{S}(0)=0.0010, \mathrm{I}(0)=0.5140, \mathrm{Z}(0)=0.3600$

3. $\mathrm{S}(0)=2.5485, \mathrm{I}(0)=0.0540, \mathrm{Z}(0)=0.8675$,

4. $\mathrm{S}(0)=0.8000, \mathrm{I}(0)=0.3154, \mathrm{Z}(0)=0.0250$.

(a) Using the parameters: $\beta=0.05, \mu=0.34, \gamma=0.015, \alpha=0.0125, \theta=0.333, B=2, \eta=0,023$. Here $\mathcal{R}_{0}=$ $0.0720156<1$. We see from Fig. 1(a) that the number of susceptibles to the meme increases as a function of time to approach the value of $S_{0}$ for the four sets of initial conditions. While Fig. 1(b,c) show that the number of spreaders and stiflers decreases as a function of time and approaches zero. Thus, for all sets of initial conditions the solution curves tend to the meme free equilibrium $E_{0}$. Hence, system (1) is locally asymptotically stable about $E_{0}$ for the above set of parameters.

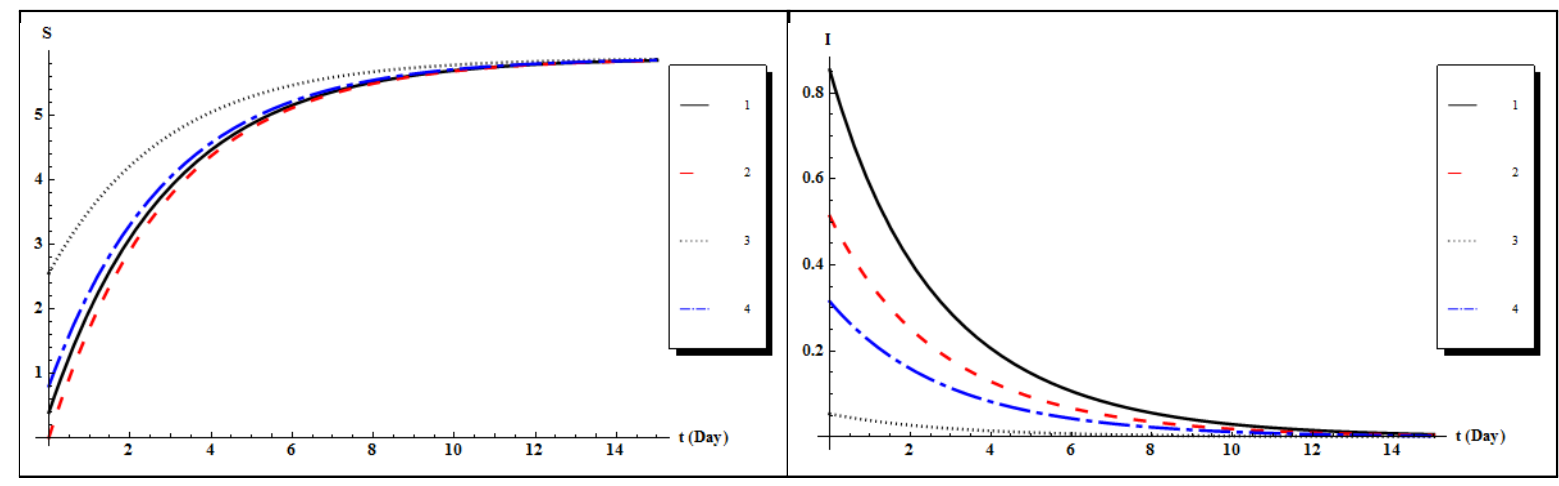

(a)

(b) 


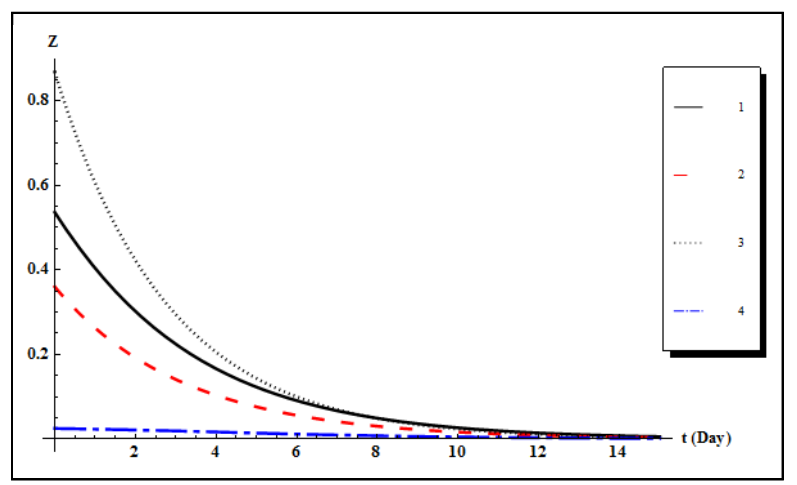

(c)

Fig. 1: Time plots of system (1) with different initial conditions for $R_{0}<1$ : (a) Susceptibles; (b) Spreaders;(c) Stiflers.

(b) Using the same parameters as in (a) except for: $\alpha=0.4, \theta=0.6, \eta=0.006$. Here $\mathcal{R}_{0}=4.15225>1$, with the same initial values. We see from Fig. 2(a) that for the second value of parameters the number of susceptibles increases first then it starts to decrease and all solutions approach a certain value $S^{*}$. Fig. 2(b) shows that the number of spreaders at the beginning of the meme increases until it approaches a certain value $I^{*}$. Fig. 2(c) shows a similar behavior for the stiflers, they grow at first then they approach a certain value $Z^{*}$. Thus, for all sets of initial conditions the solution curves tend to the positive(meme existence) equilibrium $E^{*}$. Hence, system (1) is locally asymptotically stable about $E^{*}$ for the above set of parameters.
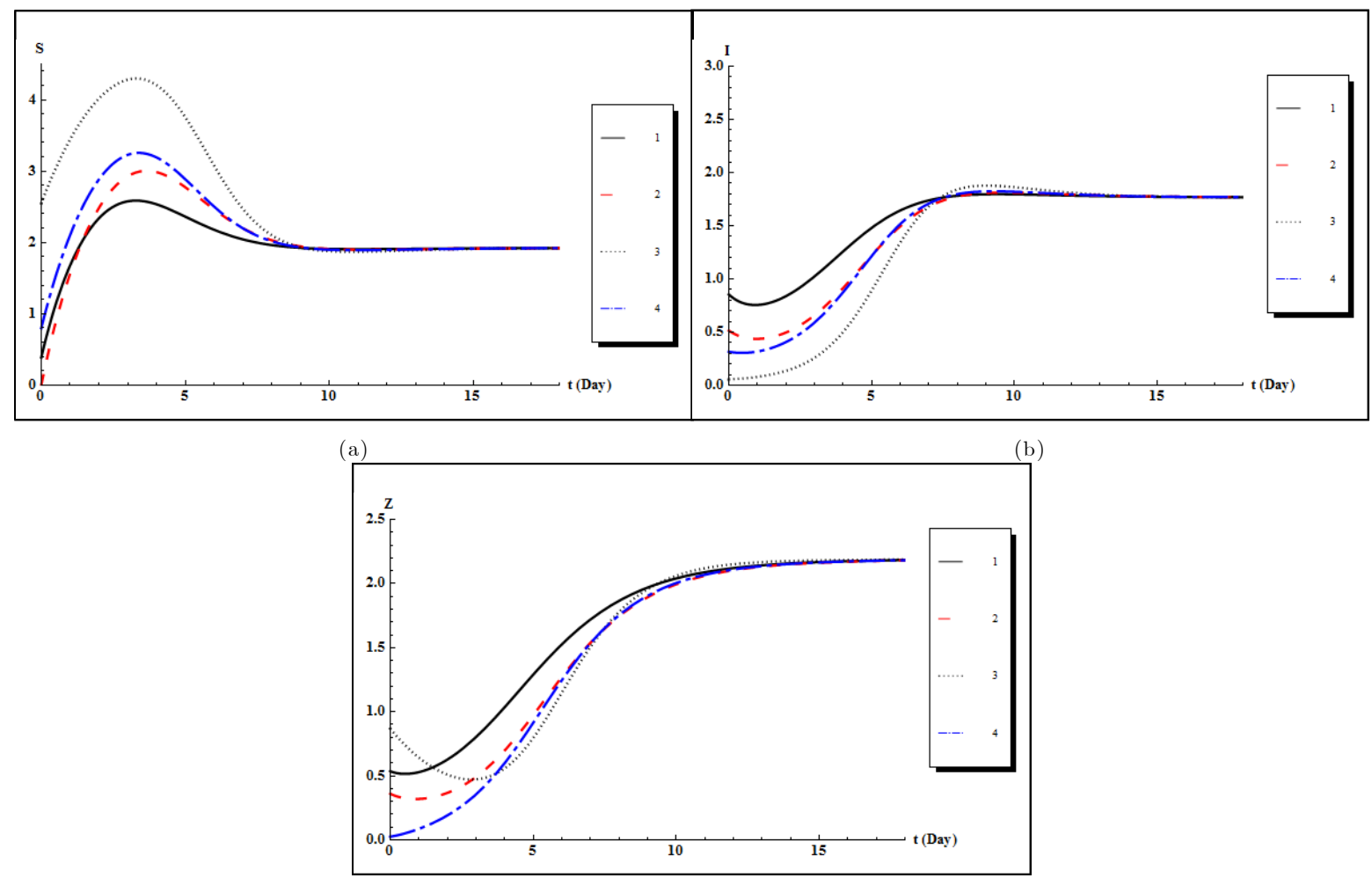

(c)

Fig. 2: Time plots of system (1) with different initial conditions for $R_{0}>1$ : (a) Susceptibles; (b) Spreaders; (c) Stiflers.

\section{Discussions and Conclusions}

In this paper, a nonlinear mathematical model of variable meme transmission is analyzed. Sufficient conditions have been given ensuring local and global stability of the meme free equilibrium point and the positive (meme existence) equilibrium point. We have shown that the model has a threshold values $R_{0}$. The meme free equilibrium $E_{0}$ is shown to be locally asymptotically stable whenever the basic reproduction number $R_{0}$ for the model is less than unity. If in addition the condition $\frac{\alpha B}{\mu^{2}} \leq 1$ is satisfied, then $E_{0}$ becomes globally asymptotically stable and the meme will 
disappear. The positive (meme existence) equilibrium $E^{*}$, if it exists, is shown to be locally asymptotically stable when $\beta>\gamma$ and $\gamma \mu>\eta \alpha \theta$. We also proved that $E^{*}$ is globally stable which means that the meme persists. Finally, some numerical simulations are used to support these results.

Although one may think that memes persist more as a result of variable meme transmission model. However, comparison between the variable meme transmission model (Model 2) presented in this paper and the constant meme transmission model (Model 1) given in [7], show no significant changes. Under the same parameters and initial conditions given in section 5, we notice in Fig. 3 and Fig. 4 that there is no difference in the behavior of solutions of the models near $E_{0}$ and $E^{*}$ respectively. All solutions approach the equilibrium points at the same time in a similar manner.
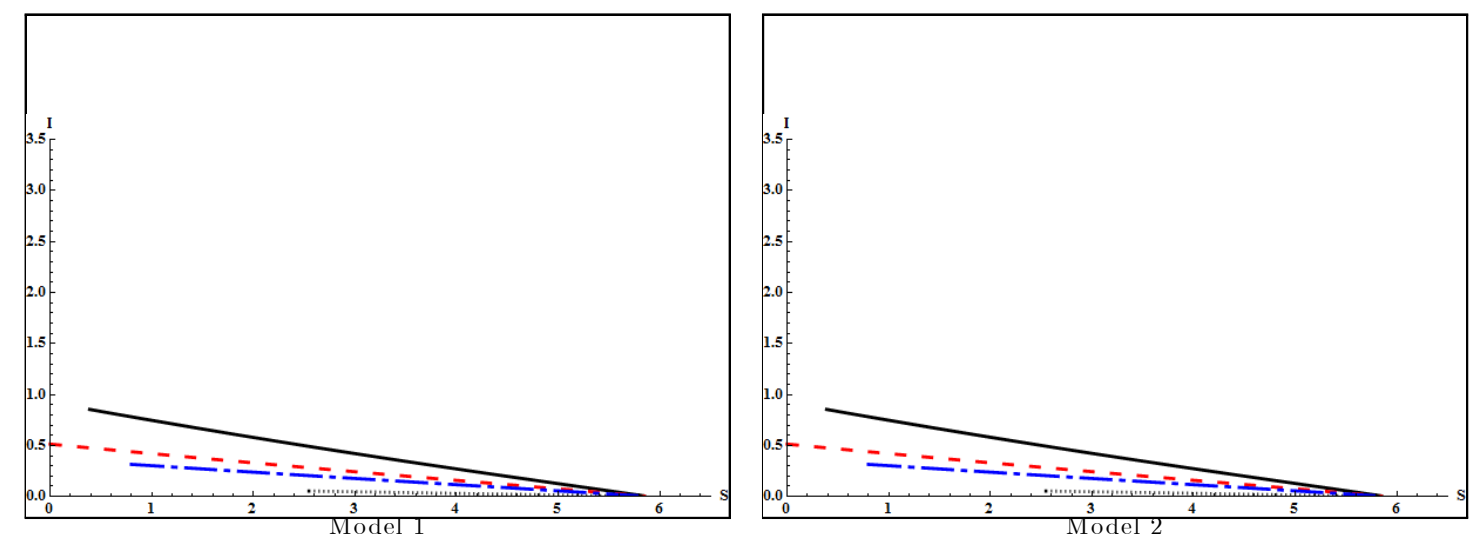

Fig. 3(a): Graphs of $S$ (susceptibles) vs. $I$ (spreaders) near $E_{0}$.
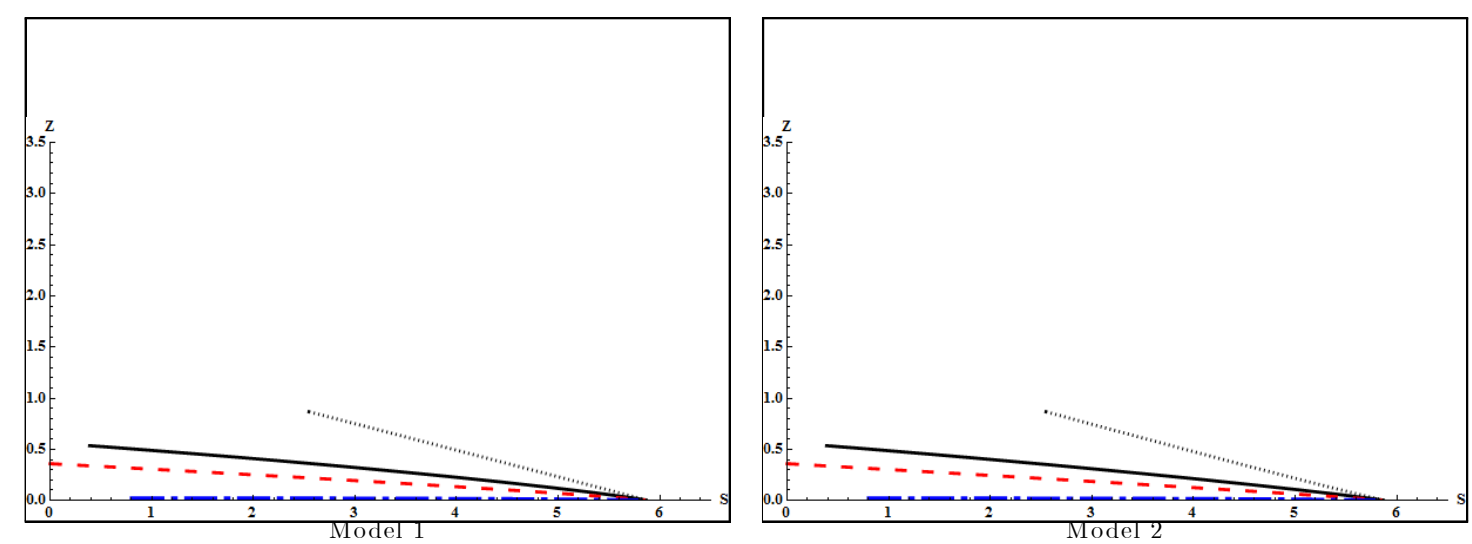

Fig. 3(b): Graphs of $S$ (susceptibles) vs. $Z$ (stiflers) near $E_{0}$.
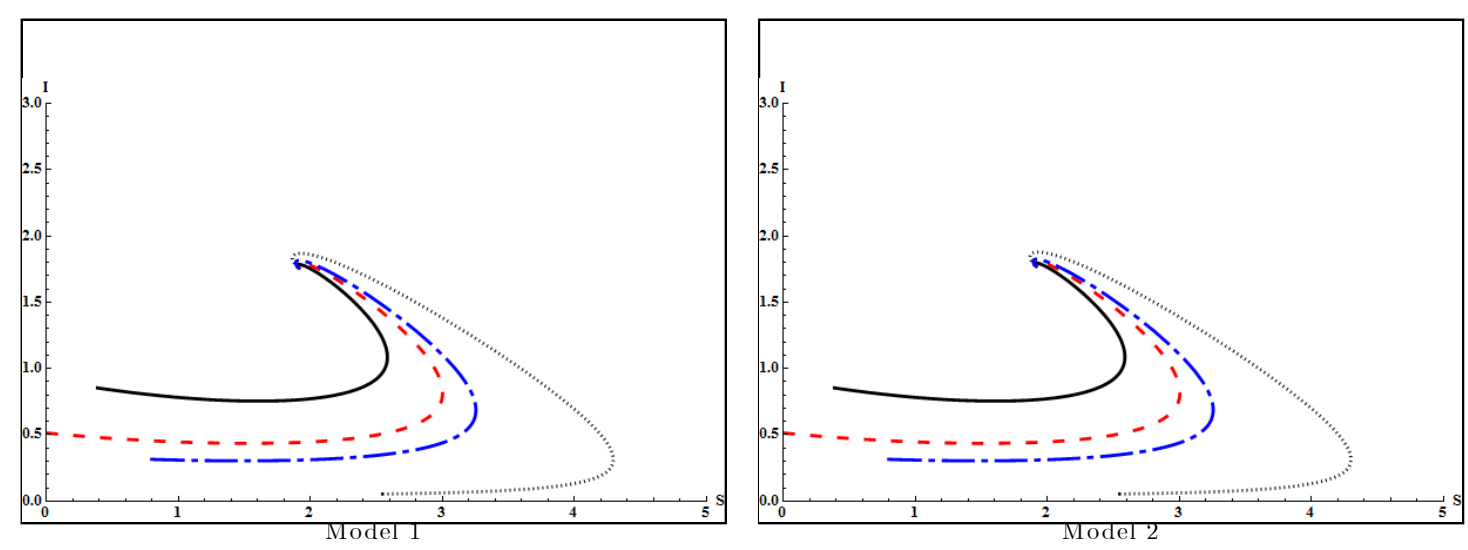

Fig. 4(a): Graphs of $S$ (susceptibles) vs. $I$ (spreaders) near $E^{*}$. 

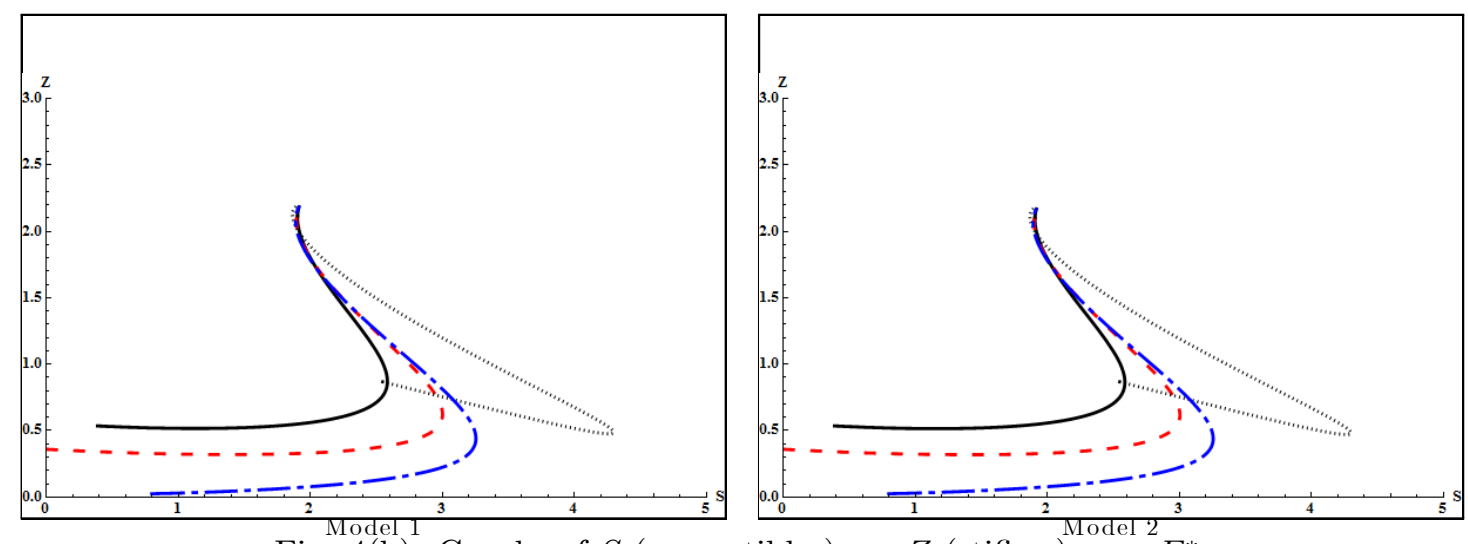

Fig. 4(b): Graphs of $S$ (susceptibles) vs. $Z$ (stiflers) near $E^{*}$.

This model may be extended in many ways to study different relations between the classes like for example, we may study the effect of stiflers on susceptibles in trying to eliminate a meme transmission.

\section{References}

[1] R. Dawkins, The Selfish Gene, 2d ed., Oxford University Press (1989).

[2] K. Dietz, Epidemics and Rumors: A survey, Journal of the Royal Statistical Society, Series A (General), Vol. 130, No.4, (1967) 505-528.

[3] V. Cane, A Note on the size of epidemics and the number of people hearing a rumor, Journal of the Royal Statistical Society, Series B (Methodological), Vol. 28, No. 3, (1966) 487-490.

[4] K. Thompson, R. Estrada, D. Daugherty, and A. Cintron-Arias, A deterministic approach to the spread of rumors, Working paper, Washington, DC, USA (2003).

[5] L. Bettencourt, A. Cintron-Arias, D. Kaiser, and C. Castillo-Chavez, The power of a good idea: Quantitative modeling of the spread of ideas from epidemiological models, Physica A, Vol. 364 (2006) 513-536.

[6] K. Kawachi, Deterministic models for rumors transmission, Nonlinear analysis: Real world applications, Vol. 9 (2008) 1989-2028.

[7] R. Al-Amoudi, S. Al-Sheikh, and S. Al-Tuwairqi, Qualitative Behavior of Solutions to a Mathematical Model of Memes Transmission, International Journal of Applied Mathematical Research, Vol. 3, No. 1 (2014) 36-44.

[8] K. Kawachi, M. Seki, H. Yoshida, Y. Otake, k. Warashina and H. Ueda, A rumor transmission model with various contact interactions, Journal of theoretical biology, Vol. 253 (2008) 55-60.

[9] J. Piqueira, Rumor propagation model: an equilibrium study, Hindawi Publishing Corporation Mathematical Problems in Engineering, Vol. (2010), Article ID 631357, doi:10.1155/2010/631357.

[10] W. Huang, On rumor spreading with skepticism and denial, Working paper (2011).

[11] L. Wang, B. Wood, An epidemiological approach to model the viral propagation of memes, Applied Mathematical Modelling, Vol. 35 (2011) 5442-5447.

[12] L. an Huo, P.Huang, and C.X. Guo, Analyzing the dynamics of a rumor transmission model with incubation, Hindawi Publishing Corporation Discrete Dynamics in Nature and Society, Vol. (2012), Article ID:328151, doi: $10.1155 / 2012 / 328151$.

[13] L. Zhao, Q. Wang, J. Cheng, Y. Chen, J. Wang, and W. Huang, Rumor spreading model with consideration of forgetting mechanism: A case of online blogging LiveJournal, Physica A, Vol. 390 (2011) 2619-2625.

[14] L. Zhao, X. Wang, X. Qiu, and J. Wang, A model for the spread of rumors in Barrat-Barthelemy-Vespignani (BBV) networks, Physica A, Vol. 392 (2013) 5542-5551. 
[15] F.Brauer, P.van den Driessche, and J.Wu, Mathematical Epidemiology, Springer-Verlag (2008).

[16] L.Perko, Differential Equations and Dynamic Systems, Springer Verlag (1991).

[17] L.Edelstein- Keshet, Mathematical Models in Biology, SIAM (2005).

[18] M.W.Hirsch, S.Smale, and R.L.Devaney, Differential equations, dynamical systems and an introduction to chaos, Elsevier Academic press (1974). 